\title{
A realization of the Lie algebra associated to a Kantor triple system
}

\author{
Jakob Palmkvist ${ }^{a)}$ \\ Albert-Einstein-Institut, Max-Planck-Institut für Gravitationsphysik, Am Mühlenberg 1, \\ D-14476 Golm, Germany
}

(Received 8 December 2005; accepted 12 December 2005;

published online 27 February 2006)

\begin{abstract}
We present a nonlinear realization of the 5-graded Lie algebra associated to a Kantor triple system. Any simple Lie algebra can be realized in this way, starting from an arbitrary 5-grading. In particular, we get a unified realization of the exceptional Lie algebras $\mathfrak{f}_{4}, \mathfrak{e}_{6}, \mathfrak{e}_{7}, \mathfrak{e}_{8}$, in which they are respectively related to the division algebras $\mathbb{R}, \mathrm{C}, \mathrm{H}, \mathrm{O}$. () 2006 American Institute of Physics.
\end{abstract}

[DOI: $10.1063 / 1.2168690]$

\section{INTRODUCTION}

The product in an associative but noncommutative algebra can be decomposed into one symmetric part, leading to a Jordan algebra, and one antisymmetric part, leading to a Lie algebra. A deeper relationship between these two important kinds of algebras is suggested by the KantorKoecher-Tits construction, ${ }^{1-3}$ which associates a Lie algebra to any Jordan algebra, and it becomes more evident when generalizing Jordan algebras to Jordan triple systems (JTS). These can further be generalized to Kantor triple systems (KTS).

The Lie algebra associated to a Jordan algebra or a JTS is 3-graded, written $\mathfrak{g}_{-1}+\mathfrak{g}_{0}+\mathfrak{g}_{1}$ as a direct sum of subspaces, while the Lie algebra associated to a KTS is 5-graded, written $\mathfrak{g}_{-2}$ $+\mathfrak{g}_{-1}+\mathfrak{g}_{0}+\mathfrak{g}_{1}+\mathfrak{g}_{2}$. We will discuss graded Lie algebras more in the following section. In Sec. III we will describe how triple systems may be obtained from graded Lie algebras and conversely construct the graded Lie algebras associated to these triple systems. Under certain conditions, we get back the original algebra, together with a nonlinear realization.

In Sec. III A we will consider Jordan triple systems and the associated 3-graded Lie algebras. In this case, the realization of the Lie algebra is said to be conformal. The operators act on $\mathfrak{g}_{-1}$ and are each either constant, linear or quadratic, according to the 3-grading. In the case of $\mathfrak{s o}(2, d)$ we get the well-known realization of the conformal algebra in $d$ dimensions, where the elements in the algebra are regarded as generators of translations (constant), Lorentz transformations together with dilatations (linear) and special conformal transformations (quadratic).

The main result of this paper, to be presented in Sec. III B, is a corresponding realization of the 5-graded Lie algebra associated to a Kantor triple system. This Lie algebra has earlier been defined as a special case of a Kantor algebra, ${ }^{4}$ using a functor that associates a Lie algebra to any generalized Jordan triple system. ${ }^{5}$ It has also been defined in a simpler but rather abstract way, as a direct sum of vector spaces together with the appropriate commutation relations. ${ }^{6}$

In our construction, the Lie algebra associated to a KTS consists of nonlinear operators acting on an extension of the KTS. The bracket arises naturally when we regard the operators as vector fields, which we will explain in Sec. II B. To our knowledge, such a construction has not appeared before. However, the concomitant realization of any simple 5-graded Lie algebra on its subspace $\mathfrak{g}_{-2}+\mathfrak{g}_{-1}$ has been obtained in Ref. 7, using a general formula for the Lie algebra of a homogeneous space.

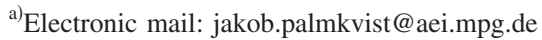


The corresponding realization of the Lie algebra associated to a Freudenthal triple system (FTS) was given in Ref. 8, called quasiconformal, and led us to the present work. The difference is that our realization is based on an arbitrary 5-grading, while in Ref. 8 the subspaces $\mathfrak{g}_{ \pm 2}$ must be one dimensional. The connection between these two realizations will be clarified in Sec. III C. As an example of interesting cases where the subspaces $\mathfrak{g}_{ \pm 2}$ are not one dimensional, we will in Sec. IV show how the exceptional Lie algebras $\mathfrak{f}_{4}, \mathfrak{e}_{6}, \mathfrak{e}_{7}, \mathfrak{e}_{8}$ can be given 5-gradings related to the division algebras $\mathbb{R}, \mathrm{C}, \mathbb{H}, O$, respectively. This construction, given in Ref. 6, together with our main result, leads to a unified realization of these exceptional Lie algebras.

\section{GRADED LIE ALGEBRAS}

We start with some definitions concerning graded Lie algebras in general, after which we will consider the cases of semisimple and simple algebras.

A Lie algebra $\mathfrak{g}$ is graded if it is the direct sum of subspaces $\mathfrak{g}_{k} \subset \mathfrak{g}$ for all integers $k$, such that

$$
\left[\mathfrak{g}_{i}, \mathfrak{g}_{j}\right] \subseteq \mathfrak{g}_{i+j}
$$

for all integers $i, j$. It is $(2 \nu+1)$-graded for some integer $\nu \geqslant 1$ if $\mathfrak{g}_{ \pm \nu} \neq 0$ and

$$
|k|>\nu \Rightarrow \mathfrak{g}_{k}=0 .
$$

(If $\mathfrak{g}_{k}=0$ for all $k \neq 0$, then $\mathfrak{g}$ will not be regarded as a graded Lie algebra.) The grade $k$ of an element $x \in \mathfrak{g}_{k}$ may be measured by a characteristic element $Z \in \mathfrak{g}$, satisfying

$$
x \in \mathfrak{g}_{k} \Rightarrow[Z, x]=k x
$$

for all integers $k$. A graded involution $\tau$ on $\mathfrak{g}$ is an automorphism of $\mathfrak{g}$ such that $\tau(\tau(x))=x$ for all $x \in \mathfrak{g}$ and $\tau\left(\mathfrak{g}_{k}\right)=\mathfrak{g}_{-k}$ for all integers $k$. If we instead of the last condition have $\tau\left(\mathfrak{g}_{k}\right)=(-1)^{k} \mathfrak{g}_{-k}$, then $\tau$ will be called a graded pseudoinvolution.

\section{A. Semisimple algebras}

Let the graded Lie algebra $\mathfrak{g}$ be semisimple, complex, and finite dimensional. Then $\mathfrak{g}$ has a unique characteristic element $Z$ that belongs to a Cartan subalgebra of $\mathfrak{g}$ contained in $\mathfrak{g}_{0}$. With respect to this Cartan subalgebra, the subspaces $\mathfrak{g}_{k}$ with $k \neq 0$ are spanned by step operators $E^{\alpha}$ corresponding to roots $\alpha$ such that

$$
E^{\alpha} \in \mathfrak{g}_{k} \Leftrightarrow E^{-\alpha} \in \mathfrak{g}_{-k},
$$

while $\mathfrak{g}_{0}$ is spanned by the Cartan elements $H^{i}$ and the remaining step operators. It follows that $\mathfrak{g}$ is $(2 \nu+1)$-graded for some integer $\nu \geqslant 1$ and the Chevalley involution

$$
E^{ \pm \alpha} \mapsto-E^{\mp \alpha}, \quad H^{i} \mapsto-H^{i}
$$

is a graded involution on $\mathfrak{g}$. Not all real forms of $\mathfrak{g}$ inherit the grading, since these are spanned by complex linear combinations of the step operators and the Cartan elements. In particular, the compact form of $\mathfrak{g}$ cannot be graded.

If we expand a root $\beta$ in the basis of simple roots $\alpha_{j}$ as $\beta=\beta^{j} \alpha_{j}$, then any set of simple roots $\alpha_{i_{1}}, \alpha_{i_{2}}, \ldots, \alpha_{i_{n}}$ generates a grading of $\mathfrak{g}$ where $\mathfrak{g}_{k}$ is spanned by all step operators $E^{\beta}$ such that $\beta^{i_{1}}+\beta^{i_{2}}+\cdots+\beta^{i_{n}}=k$ and, if $k=0$, the Cartan elements. Any 3-grading or 5-grading of a simple Lie algebra can be obtained in this way (possibly after an automorphism). If $\mathfrak{g}$ is simple and 3-graded or 5-graded, we also have $\left[\mathfrak{g}_{i}, \mathfrak{g}_{j}\right]=\mathfrak{g}_{i+j}$ for $i, j= \pm 1$ and (up to an automorphism) there is a unique 5-grading with one dimensional subspaces $\mathfrak{g}_{ \pm 2}$, except for $\mathfrak{g}=\mathfrak{a}_{1}$. On the other hand, $\mathfrak{e}_{8}, \mathfrak{f}_{4}, \mathfrak{g}_{2}$ cannot be 3-graded. A table of all simple 3-graded and 5-graded Lie algebras can be found in Ref. 9. 


\section{B. Algebras of operators}

We will now describe how any vector space $U$ or pair of vector spaces $V, W$ gives rise to an infinite dimensional graded Lie algebra $T(U)$ or $T(V, W)$ consisting of operators acting on $U$ or $V \oplus W$.

With an operator $f$ on a vector space $U$ we mean a map $U \rightarrow U$. It is of order $p \geqslant 1$ if there is a symmetric $p$-linear map $F: U^{p} \rightarrow U$ such that

$$
f(u)=F(u, \ldots, u)
$$

for all $u \in U$, and of order 0 if there is a vector $v \in U$ such that $f(u)=v$ for all $u \in U$. We define the composition of $f$ and another operator $g$ on $U$ by

$$
(f \circ g)(u)=p F(g(u), u, \ldots, u)
$$

or $f \circ g=0$ if $f$ is of order 0 .

For any integer $k \geqslant-1$, let $T_{k}(U)$ be the vector space consisting of all operators on $U$ of order $k+1$. Furthermore, set $T_{k}(U)=0$ for all integers $k \leqslant-2$ and let $T(U)$ be the direct sum of all these vector spaces. Now $T(U)$, together with the bracket

$$
[f, g]=f \circ g-g \circ f,
$$

is a graded Lie algebra, isomorphic to the algebra of all vector fields $f^{i} \partial_{i}$ on $U$ such that $f$ $\in T(U)$. The isomorphism is given by $f \mapsto-f^{i} \partial_{i}$.

Similarly, for any pair of vector spaces $V, W$, we can define a graded Lie algebra $T(V, W)$ of operators on $V \oplus W$, isomorphic to the algebra of all vector fields $f^{i} \partial_{i}$ on $V \oplus W$ such that $f$ $\in T(V, W)$. As a graded Lie algebra, $T(V, W)$ is the direct sum of subspaces $T_{k}(V, W)$ for all integers $k$, where $T_{k}(V, W)=0$ for $k \leqslant-3$.

With a realization of a Lie algebra $\mathfrak{g}$ on $U$ or $V \oplus W$ we mean a homomorphism from $\mathfrak{g}$ to $T(U)$ or $T(V, W)$. If all elements are mapped on linear operators, it reduces to a linear representation. In the following section, we will see that any simple 3-graded or 5-graded Lie algebra $\mathfrak{g}$ can be described as a subalgebra of $T\left(\mathfrak{g}_{-1}\right)$ or $T\left(\mathfrak{g}_{-1}, \mathfrak{g}_{-2}\right)$ and this description will thus give us a realization of the algebra.

\section{TRIPLE SYSTEMS}

In this section, we will clarify the connection between graded Lie algebras and triple systems. Jordan triple systems and Kantor triple systems correspond to general 3-graded and 5-graded algebras, respectively, while Freudenthal triple systems correspond to 5-graded algebras with one dimensional subspaces $\mathfrak{g}_{ \pm 2}$.

A triple system (or ternary algebra) is a vector space $U$ together with a linear map

$$
U \times U \times U \rightarrow U, \quad(x, y, z) \mapsto(x y z)
$$

called triple product. For any two elements $u, v$ in a triple system $U$, we define the linear operator $\langle u, v\rangle$ on $U$ by

$$
\langle u, v\rangle(z)=(u z v)-(v z u) .
$$

Let $\mathfrak{g}$ be a graded Lie algebra with a graded involution $\tau$. Then the vector space $\mathfrak{g}_{-1}$ together with the triple product

$$
(x y z)=[[x, \tau(y)], z]
$$

is a triple system, which will be called the triple system derived from $\mathfrak{g}$. We have the identity

$$
(u v(x y z))-(x y(u v z))=((u v x) y z)-(x(v u y) z)
$$

from the fact that $\tau$ is an involution and from the Jacobi identity, which also gives us 


$$
\langle u, v\rangle(z)=[[u, v], \tau(z)]
$$

for all $u, v, z \in \mathfrak{g}_{-1}$.

\section{A. Jordan triple systems}

Let $\mathfrak{g}$ be a 3 -graded Lie algebra with a graded involution. Since $[u, v]=0$ for any $u, v \in \mathfrak{g}_{-1}$ we have

$$
\langle u, v\rangle(z)=0
$$

in the triple system derived from $\mathfrak{g}$, which means that the triple product $(u z v)$ is symmetric in $u$ and $v$.

We define a Jordan triple system (JTS) ${ }^{10}$ as a triple system where the identities (3.1) and (3.2) hold. Thus the triple system derived from a 3-graded Lie algebra with a graded involution is a JTS. Conversely, any Jordan triple system $J$ gives rise to a 3-graded subalgebra of $T(J)$, spanned by the operators

$$
\begin{gathered}
u_{a}(x)=a, \\
s_{a b}(x)=(a b x), \\
\tilde{u}_{a}(x)=-\frac{1}{2}(x a x),
\end{gathered}
$$

where $a, b, x \in J$. This is the Lie algebra $L(J)$ associated to the Jordan triple system $J$. From (3.1) and (3.2) we get the commutation relations

$$
\begin{gathered}
{\left[s_{a b}, s_{c d}\right]=s_{(a b c) d}-s_{c(b a d)}, \quad\left[s_{a b}, u_{c}\right]=u_{(a b c)},} \\
{\left[s_{a b}, \tilde{u}_{c}\right]=-\tilde{u}_{(b a c)}, \quad\left[u_{a}, \tilde{u}_{b}\right]=s_{a b},}
\end{gathered}
$$

and $\left[\tilde{u}_{a}, \tilde{u}_{b}\right]=0$. [We also have $\left[u_{a}, u_{b}\right]=0$ already from the definition of $T(J)$.] It follows that if $J$ is derived from a simple 3 -graded Lie algebra $\mathfrak{g}$ with a graded involution $\tau$, then $\mathfrak{g}$ is isomorphic to $L(J)$ with the isomorphism

$$
\begin{array}{r|ccc}
+1 & \tau(a) & \mapsto & \tilde{u}_{a} \\
0 & {[a, \tau(b)]} & \mapsto & {\left[u_{a}, \tilde{u}_{b}\right]} \\
-1 & a & \mapsto & u_{a}
\end{array}
$$

where $a, b \in \mathfrak{g}_{-1}$. This is the conformal realization of $\mathfrak{g}$ on $\mathfrak{g}_{-1}$.

\section{B. Kantor triple systems}

If $\mathfrak{g}$ is a 5-graded Lie algebra with a graded involution, then the identity

$$
(u v(x y z))-(x y(u v z))=((u v x) y z)-(x(v u y) z)
$$

still holds in the triple system derived from $\mathfrak{g}$ but instead of $\langle u, v\rangle=0$ we now have the identity

$$
\langle\langle u, v\rangle(x), y\rangle=\langle(y x u), v\rangle-\langle(y x v), u\rangle .
$$

We define a Kantor triple system (KTS), ${ }^{11}$ or a JTS of second order $^{5}$ as a triple system such that (3.4) and (3.5) hold. Thus the triple system derived from a 5-graded Lie algebra with a graded involution is a KTS, and so is any JTS.

Let $K$ be a KTS and let $L$ be the vector space spanned by all linear operators $\langle u, v\rangle$ on $K$, where $u, v \in K$. If $K$ is derived from a simple 5-graded Lie algebra $\mathfrak{g}$ with a graded involution $\tau$, 
then we can identify not only $K$ with $\mathfrak{g}_{-1}$, but also $L$ with $\mathfrak{g}_{-2}$ by $\langle u, v\rangle=[u, v]$. In analogy with the construction of $L(J)$ in the preceding section we can now construct a 5 -graded subalgebra of $T(K, L)$ spanned by the operators

$$
\begin{gathered}
K_{a b}(z+Z)=2\langle a, b\rangle, \\
U_{a}(z+Z)=a+\langle a, z\rangle, \\
S_{a b}(z+Z)=(a b z)-\langle a, Z(b)\rangle, \\
\tilde{U}_{a}(z+Z)=-\frac{1}{2}(z a z)-\frac{1}{2} Z(a)+\frac{1}{6}\langle(z a z), z\rangle-\frac{1}{2}\langle Z(a), z\rangle, \\
\tilde{K}_{a b}(z+Z)=-\frac{1}{6}(z\langle a, b\rangle(z) z)-\frac{1}{2} Z(\langle a, b\rangle(z))+\frac{1}{12}\langle(z\langle a, b\rangle(z) z), z\rangle+\frac{1}{2}\langle Z(a), Z(b)\rangle,
\end{gathered}
$$

where $a, b, z \in K$ and $Z \in L$. This is the Lie algebra $L(K)$ associated to the Kantor triple system $K$. We get the commutation relations

$$
\begin{gathered}
{\left[S_{a b}, S_{c d}\right]=S_{(a b c) d}-S_{c(b a d)}, \quad\left[S_{a b}, U_{c}\right]=U_{(a b c)},} \\
{\left[S_{a b}, K_{c d}\right]=K_{\langle c, d\rangle(b) a}, \quad\left[U_{a}, U_{b}\right]=K_{a b},} \\
{\left[S_{a b}, \widetilde{U}_{c}\right]=-\widetilde{U}_{(b a c)}, \quad\left[S_{a b}, \widetilde{K}_{c d}\right]=-\widetilde{K}_{\langle c, d\rangle(a) b},} \\
{\left[U_{a}, \tilde{U}_{b}\right]=S_{a b}, \quad\left[U_{a}, \widetilde{K}_{c d}\right]=-\widetilde{U}_{\langle c, d\rangle(a)},} \\
{\left[K_{a b}, \widetilde{U}_{c}\right]=U_{\langle a, b\rangle(c)}, \quad\left[K_{a b}, \widetilde{K}_{c d}\right]=S_{\langle a, b\rangle(c) d}-S_{\langle a, b\rangle(d) c},} \\
{\left[\widetilde{U}_{a}, \widetilde{U}_{b}\right]=\widetilde{K}_{a b}, \quad\left[\widetilde{K}_{a b}, \widetilde{K}_{c d}\right]=\left[\widetilde{K}_{a b}, \widetilde{U}_{c}\right]=0 .}
\end{gathered}
$$

It follows that if $K$ is derived from a simple 5-graded Lie algebra $\mathfrak{g}$ with a graded involution $\tau$, then $\mathfrak{g}$ is isomorphic to $L(K)$ with the isomorphism

$$
\begin{array}{r|ccc}
+2 & {[\tau(a), \tau(b)]} & \mapsto\left[\tilde{U}_{a}, \widetilde{U}_{b}\right]=\widetilde{K}_{a b} \\
+1 & \tau(a) & \mapsto \quad \tilde{U}_{a} \\
0 & {[a, \tau(b)]} & \mapsto\left[U_{a}, \widetilde{U}_{b}\right]=S_{a b} \\
-1 & a & \mapsto \quad U_{a} \\
-2 & {[a, b]} & \mapsto\left[U_{a}, U_{b}\right]=K_{a b}
\end{array}
$$

where $a, b \in \mathfrak{g}_{-1}$. Then this isomorphism will be a realization of $\mathfrak{g}$ on its subspace $\mathfrak{g}_{-2}+\mathfrak{g}_{-1}$. The Lie algebra associated to a Kantor triple system can also be defined by the commutation relations above, and this is partly the definition given in Refs. 6 and 12, but it does not directly lead to a realization like (3.6). On the other hand, with our construction, we have to derive the commutation relations from the definition of the operators and the defining properties of a Kantor triple system. This requires long calculations and we will only give a few of them here. The full expressions are written out in Ref. 13. As an example, we have 


$$
\begin{aligned}
{\left[U_{a}, \widetilde{U}_{b}\right](z+Z)=} & \left\langle a,-\frac{1}{2}(z b z)-\frac{1}{2} Z(b)\right\rangle \\
& +\frac{1}{2}(a b z)+\frac{1}{2}(z b a)+\frac{1}{2}\langle a, z\rangle(b) \\
& -\frac{1}{6}\langle(a b z), z\rangle-\frac{1}{6}\langle(z b a), z\rangle-\frac{1}{6}\langle(z b z), a\rangle \\
& +\frac{1}{2}\langle\langle a, z\rangle(b), z\rangle+\frac{1}{2}\langle Z(b), a\rangle \\
= & (a b z)+\langle Z(b), a\rangle+\frac{3}{6}\langle\langle a, z\rangle(b), z\rangle \\
& -\frac{1}{6}\langle(z b a), z\rangle-\frac{1}{6}\langle(a b z), z\rangle+\frac{2}{6}\langle(z b z), a\rangle \\
= & (a b z)+\langle Z(b), a\rangle=S_{a b}(z+Z),
\end{aligned}
$$

where we have used

$$
\begin{aligned}
3\langle\langle a, z\rangle(b), z\rangle= & 2\langle\langle a, z\rangle(b), z\rangle+\langle\langle a, z\rangle(b), z\rangle \\
= & 2(\langle(z b a), z\rangle-2\langle(z b z), a\rangle) \\
& +\langle(a b z), z\rangle-\langle(z b a), z\rangle \\
= & \langle(z b a), z\rangle+\langle(a b z), z\rangle-2\langle(z b z), a\rangle .
\end{aligned}
$$

Among the other commutators, $\left[U_{a}, U_{b}\right]$ and $\left[S_{a b}, U_{c}\right]$ are easy to calculate, while $\left[\widetilde{U}_{a}, \widetilde{U}_{b}\right]$ and $\left[S_{a b}, \widetilde{U}_{c}\right]$ are much harder. It is convenient to first verify the identities

$$
\begin{gathered}
\{((z b z) a z)+2(z a(z b z))\}_{a b}=(z\langle b, a\rangle(z) z), \\
\{(\langle x, y\rangle(b) a z)\}_{a b}=(x\langle a, b\rangle(y) z)-(y\langle a, b\rangle(x) z),
\end{gathered}
$$

where we denote antisymmetrization by curly brackets, $\{f(a, b)\}_{a b}=f(a, b)-f(b, a)$ for any function $f$. We can also use (3.9) to rewrite the last term in $\widetilde{K}_{a b}(z+Z)$ and show that the map (3.7) is well defined in the sense that $\widetilde{K}_{a b}=\widetilde{K}_{c d}$ if $[a, b]=[c, d]$. It turns out that

$$
\begin{aligned}
2\langle\langle u, v\rangle(a),\langle x, y\rangle(b)\rangle= & \langle(x\langle a, b\rangle(y) u), v\rangle-\langle(y\langle a, b\rangle(x) u), v\rangle \\
& +\langle(y\langle a, b\rangle(x) v), u\rangle-\langle(x\langle a, b\rangle(y) v), u\rangle .
\end{aligned}
$$

The remaining nonzero commutation relations follow from the Jacobi identity. Finally, we can show that

$$
\left[\left[\tilde{K}_{a b}, \tilde{U}_{c}\right], K_{x y}\right]=\left[\left[\tilde{K}_{a b}, \widetilde{U}_{c}\right], U_{z}\right]=0
$$

which gives us

$$
\left[\tilde{K}_{a b}, \tilde{U}_{c}\right]=\left[\tilde{K}_{a b}, \tilde{K}_{c d}\right]=0 .
$$

\section{Freudenthal triple systems} product

Let $\mathfrak{g}$ be a 5 -graded Lie algebra and let $T$ be an element in $\mathfrak{g}_{2}$. Then $\mathfrak{g}_{-1}$ together with the triple

$$
(x y z)=[[x,[T, y]], z]
$$

is a triple system satisfying

$$
\langle x, y\rangle(z)=(y x z)-(x y z) .
$$

Suppose now that the subspaces $\mathfrak{g}_{ \pm 2}$ are one dimensional. If we extend the map 


$$
\mathfrak{g}_{-1} \rightarrow \mathfrak{g}_{1}, \quad x \mapsto[T, x]
$$

to a graded pseudoinvolution $\tau$ on $\mathfrak{g}$, then for any $x, y \in \mathfrak{g}_{-1}$ there is a scalar $\alpha$ such that $\langle x, y\rangle$ $\times(z)=\alpha z$. Thus we can identify the vector space spanned by all operators $\langle x, y\rangle$ where $x, y \in \mathfrak{g}_{-1}$ with the field over which the Lie algebra is defined, writing

$$
\langle x, y\rangle(z)=\langle x, y\rangle z
$$

and we can regard $\langle x, y\rangle$ as an antisymmetric bilinear form on the triple system rather than an operator. Since $\tau$ is not an involution but a pseudoinvolution, we now have the identity

$$
(u v(x y z))-(x y(u v z))=((u v x) y z)+(x(v u y) z)
$$

with a changed sign of the last term, in comparison to (3.4). However, (3.5) still holds. We define a Freudenthal triple system (FTS) as a triple system with an antisymmetric bilinear form satisfying (3.5), (3.10), and (3.12). To sum up, we have

$$
\begin{gathered}
(u v(x y z))=((u v x) y z)+(x(v u y) z)+(x y(u v z)), \\
\langle x, y\rangle z=(x z y)-(y z x)=(y x z)-(x y z), \\
\langle u, v\rangle\langle x, y\rangle=\langle(y x u), v\rangle-\langle(y x v), u\rangle .
\end{gathered}
$$

We note that (3.13) cannot be replaced by (3.4) or, in other words, that a KTS cannot satisfy (3.14) and (3.15) for some antisymmetric bilinear form (unless this is identically equal to zero, in which case the KTS reduces to a JTS).

Let $F$ be a FTS and let $L$ be the vector space spanned by all operators $\langle u, v\rangle$ on $F$ where $u, v \in F$. If we change some of the signs in the definition of $\widetilde{K}_{a b}$ in (3.6), keep the definitions of all the other operators and simplify the expressions by (3.10)-(3.12), then we get

$$
\begin{gathered}
K_{a b}(z+\zeta)=2\langle a, b\rangle, \\
U_{a}(z+\zeta)=a+\langle a, z\rangle, \\
S_{a b}(z+\zeta)=(a b z)-\zeta\langle a, b\rangle, \\
\tilde{U}_{a}(z+\zeta)=-\frac{1}{2}(z a z)-\frac{1}{2} \zeta a+\frac{1}{6}\langle(z z z), a\rangle-\frac{1}{2} \zeta\langle a, z\rangle, \\
\tilde{K}_{a b}(z+\zeta)=\frac{1}{6}\langle a, b\rangle(z z z)+\frac{1}{2} \zeta\langle a, b\rangle z-\frac{1}{12}\langle a, b\rangle\langle(z z z), z\rangle+\frac{1}{2} \zeta^{2}\langle a, b\rangle,
\end{gathered}
$$

where $a, b, z \in F$ and $\zeta \in L$. These operators span a subalgebra of $T(F, L)$ with the commutation relations

$$
\begin{gathered}
{\left[S_{a b}, S_{c d}\right]=S_{(a b c) d}+S_{c(b a d)}, \quad\left[S_{a b}, U_{c}\right]=U_{(a b c)},} \\
{\left[S_{a b}, K_{c d}\right]=\langle c, d\rangle K_{b a}, \quad\left[U_{a}, U_{b}\right]=K_{a b},} \\
{\left[S_{a b}, \widetilde{U}_{c}\right]=\widetilde{U}_{(b a c)}, \quad\left[S_{a b}, \widetilde{K}_{c d}\right]=\langle c, d\rangle \widetilde{K}_{a b},} \\
{\left[U_{a}, \widetilde{U}_{b}\right]=S_{a b}, \quad\left[U_{a}, \widetilde{K}_{c d}\right]=\langle c, d\rangle \widetilde{U}_{a},}
\end{gathered}
$$




$$
\begin{gathered}
{\left[K_{a b}, \widetilde{U}_{c}\right]=\langle a, b\rangle U_{c}, \quad\left[K_{a b}, \widetilde{K}_{c d}\right]=\langle a, b\rangle\left(S_{c d}-S_{d c}\right),} \\
{\left[\widetilde{U}_{a}, \widetilde{U}_{b}\right]=\widetilde{K}_{a b}, \quad\left[\widetilde{K}_{a b}, \widetilde{K}_{c d}\right]=\left[\widetilde{K}_{a b}, \widetilde{U}_{c}\right]=0 .}
\end{gathered}
$$

It follows that if $F$ is derived from a simple 5-graded Lie algebra $\mathfrak{g}$ with one dimensional subspaces $\mathfrak{g}_{ \pm 2}$ and a graded pseudoinvolution as described above, then the map (3.7) is again an isomorphism. This is the quasiconformal realization of $\mathfrak{g}$ on $\mathfrak{g}_{-2}+\mathfrak{g}_{-1}$, given in Ref. 8 [where the factor of -2 in (17) and the opposite sign of the bracket lead to different coefficients in (29)].

Freudenthal triple systems where the antisymmetric bilinear form is nondegenerate are in a one-to-one correspondence to simple, complex, and finite-dimensional Lie algebras. ${ }^{14}$ Since such a Lie algebra is also associated to a KTS, it follows that any nondegenerate FTS can be obtained from a KTS. Although Freudenthal triple systems are sufficient to obtain all simple finitedimensional Lie algebras, the result in the following section shows that also Kantor triple systems may be useful.

\section{EXCEPTIONAL LIE ALGEBRAS}

We end this paper with some comments on the exceptional Lie algebras $\mathfrak{f}_{4}, \mathfrak{e}_{6}, \mathfrak{e}_{7}, \mathfrak{e}_{8}$. These are associated to Kantor triple systems which in turn can be defined using the division algebras $\mathrm{R}, \mathrm{C}, \mathrm{H}, \mathrm{O}$. We will briefly describe this construction, given in Ref. 6 and extended in Ref. 12.

Let $\mathbb{K}$ be one of the division algebras $\mathbb{R}, \mathrm{C}, \mathbb{H}, \mathrm{O}$, consisting of real and complex numbers, quaternions and octonions, ${ }^{15}$ respectively. Then the tensor product algebra $\mathbb{K} \otimes 0$ is a KTS with the triple product

$$
(x y z)=x\left(y^{*} z\right)+z\left(y^{*} x\right)-y\left(x^{*} z\right),
$$

where the conjugation in $\mathbb{K} \otimes O$ is given from the conjugations in $\mathbb{K}$ and $O$ simply by

$$
(a, b)^{*}=\left(a^{*}, b^{*}\right) .
$$

The complex Lie algebras $L(\mathbb{K} \otimes 0)$ associated to these triple systems are

$$
\begin{aligned}
& L(\mathbb{R} \otimes O)=\mathfrak{f}_{4}, \\
& L(\mathbb{C} \otimes O)=\mathfrak{e}_{6}, \\
& L(\mathbb{H} \otimes O)=\mathfrak{e}_{7}, \\
& L(O \otimes O)=\mathfrak{e}_{8} .
\end{aligned}
$$

Thus we obtain 5-gradings of these algebras, but the subspaces $\mathfrak{g}_{ \pm 2}$ are not one dimensional. If we include also the split forms of $\mathrm{C}, \mathrm{H}, \mathrm{O}$ in a similar way and consider the real Lie algebras, we get all noncompact forms of $\mathfrak{f}_{4}, \mathfrak{e}_{6}, \mathfrak{e}_{7}, \mathfrak{e}_{8} .{ }^{12}$

The construction (3.6) of $L(K)$ for any Kantor triple system $K$ now leads to a unified realization of the exceptional Lie algebras $\mathfrak{f}_{4}, \mathfrak{e}_{6}, \mathfrak{e}_{7}, \mathfrak{e}_{8}$. This would be an interesting subject of further studies.

\section{ACKNOWLEDGMENTS}

The author is very grateful to Martin Cederwall for many valuable comments and suggestions. The author would also like to thank Issai Kantor for helpful explanations and providing copies of some reference articles. 
${ }^{1}$ I. L. Kantor, Sov. Math. Dokl. 5, 1404 (1964).

${ }^{2}$ M. Koecher, Am. J. Math. 89, 787 (1967).

${ }^{3}$ J. Tits, Ind. Math. 24, 530 (1962).

${ }^{4}$ H. Asano and S. Kaneyuki, Tokyo J. Math. 11, 105 (1988).

${ }^{5}$ I. L. Kantor, Trudy Sem. Vect. Tens. Anal. 16, 407 (1972).

${ }^{6}$ I. L. Kantor, Sov. Math. Dokl. 14, 254 (1973).

${ }^{7}$ I. Kantor, J. Algebra 235, 766 (2001).

${ }^{8}$ M. Günaydin, K. Koepsell, and H. Nicolai, Commun. Math. Phys. 221, 57 (2001).

${ }^{9}$ J. Faraut, S. Kaneyuki, A. Korányi, Q. Lu, and G. Roos, Analysis and Geometry on Complex Homogeneous Domains (Birkhäuser, Boston, 2000).

${ }^{10}$ N. Jacobson, Am. J. Math. 71, 149 (1949).

${ }^{11}$ B. N. Allison and J. R. Faulkner, Commun. Algebra 27, 519 (1999).

${ }^{12}$ D. Mondoc, Doctoral thesis, Lund University, 2002.

${ }^{13}$ J. Palmkvist, Master thesis, Chalmers University of Technology and Göteborg University, 2005.

${ }^{14}$ I. L. Kantor and I. M. Skopets, Sel. Math. Sov. 2, 293 (1982)

${ }^{15}$ J. C. Baez, Bull. Am. Math. Soc. 39, 145 (2002). 